
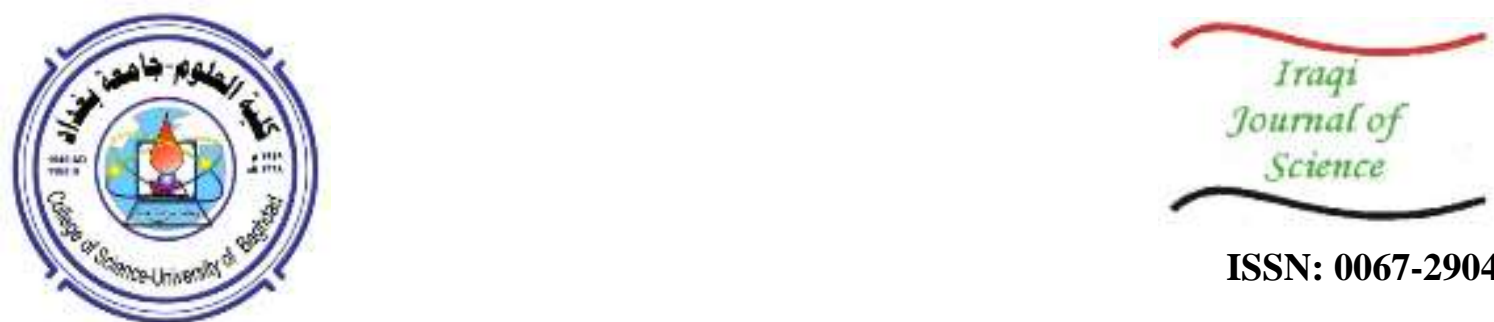

ISSN: 0067-2904

\title{
Stability And Data Dependence Results For The Mann Iteration Schemes on n-Banach Space
}

\author{
Mustafa Mohamed Hamed*, Zeana Zaki Jamil \\ Department of Mathematics, College of Science, University of Baghdad, Baghdad, Iraq
}

Received: 29/8/2019 Accepted: 22/10/2019

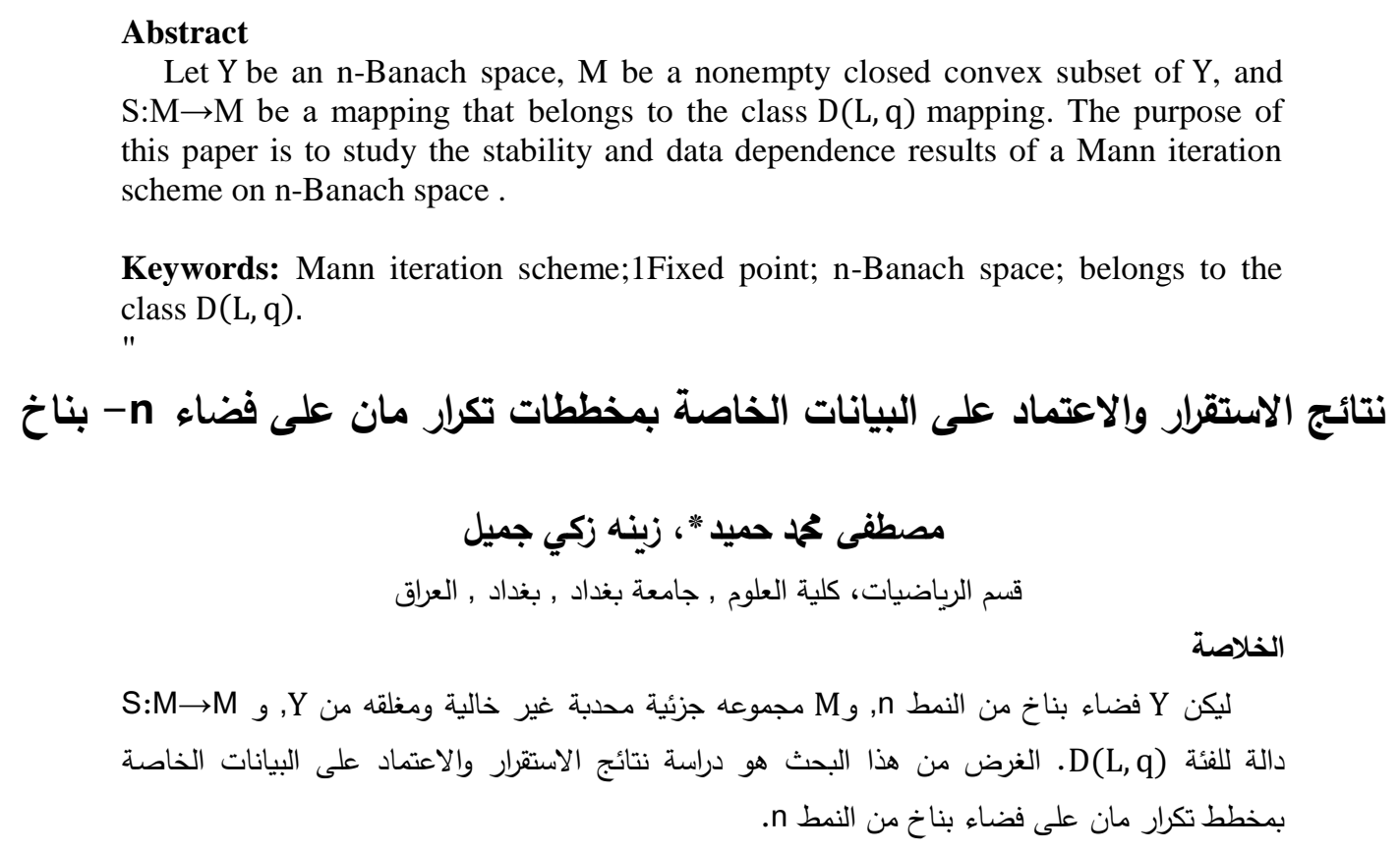

Introduction

Let $\mathrm{Y}$ be a vector space over a field $\mathbb{F}$, which is either a real or complex space with a finite dimension $d \geq n ; n \in \mathbb{N}$ or an infinite dimension, and a real-valued function $\|., \ldots\|:, Y^{\mathrm{n}} \rightarrow \mathbb{R}$ satisfying the following properties for all $\mathrm{x}, \mathrm{v}_{1}, \ldots, \mathrm{v}_{\mathrm{n}-1} \in \mathrm{Y}$ and $\lambda \in \mathbb{R}$.

1. $\left\|\mathrm{x}, \mathrm{v}_{1}, \ldots, \mathrm{v}_{\mathrm{n}-1}\right\|=0$ if and1only if $\mathrm{x}, \mathrm{v}_{1}, \ldots, \mathrm{v}_{\mathrm{n}-1}$ are linearly dependent.

2. $\left\|\mathrm{x}, \mathrm{v}_{1}, \ldots, \mathrm{v}_{\mathrm{n}-1}\right\|$ is invariantlunder permutations of $\mathrm{x}, \mathrm{v}_{1}, \ldots, \mathrm{v}_{\mathrm{n}-1}$.

3. $\left\|\lambda \mathrm{x}, \mathrm{v}_{1}, \ldots, \mathrm{v}_{\mathrm{n}-1}\right\|=|\lambda|\left\|\mathrm{x}, \mathrm{v}_{1}, \ldots, \mathrm{v}_{\mathrm{n}-1}\right\|$.

4. $\left\|x+y, v_{1}, \ldots, v_{n-1}\right\| \leq\left\|x, v_{1}, \ldots, v_{n-1}\right\|+\left\|y, v_{1}, \ldots, v_{n-1}\right\|$.

then $\|., \ldots$,$\| is called an n$-norm on $Y$, and $(Y,\|, \ldots,\|$.$) is called a linear n$-normed space. For simplicity, we will call Y an n-normed space [1]. In the following, we need the concept of $n$-Banach space. H. Gunawan and M. Mashadi [1] studied the concepts of Cauchy sequence and convergence sequence, as follows:

A sequence $\left\{x_{n}\right\}$ in $n$-normed space $(Y, \|, \ldots, \ldots)$ is said to be a converge to $x \in Y$, if $\lim _{\mathrm{n} \rightarrow \infty}\left\|\mathrm{x}_{\mathrm{n}}-\mathrm{x}, \mathrm{v}_{1}, \mathrm{v}_{2}, \ldots, \mathrm{v}_{\mathrm{n}-1}\right\|=0$ for all $\mathrm{v}_{1}, \mathrm{v}_{2}, \ldots, \mathrm{v}_{\mathrm{n}-1} \in \mathrm{Y}$,

Also, a sequence $\left\{x_{n}\right\}$ in n-normed space $(Y,\|., \ldots,\|$.$) is said to be a Cauchy sequence if$ $\lim _{m, n \rightarrow \infty}\left\|x_{n}-x_{m}, v_{1}, v_{2}, \ldots, v_{n-1}\right\|=0$ for all $v_{1}, v_{2}, \ldots, v_{n-1} \in Y$

Therefore, if every Cauchy sequence is a convergent sequence, then the $(Y,\|., \ldots,\|$.$) is called an$ n-Banach space. 
The concept of stability was introduced by Harder [2] and Harder and Hicks [3], roughly speaking of a fixed point iteration procedure which is numerically stable if a small modification in the initial data involved in the computation process will produce a small influence on the computed value of the fixed point.

The outcomes on the data dependence results for the Mann iteration on normed space using contraction mapping were proved by Solutz [4]. Also, data dependence results were discussed by many authors, including Rus and Muresan [5], Espiýnola and Petrusel [6], and Soltuz [7].

In this paper, we generalize the concept of stability on an n-normed space and we prove the stability of the Mann iteration. We establish data dependence results of the Mann iteration scheme on nBanach space, under a mapping that belongs to the class $D(L, q)$ :

Let $M$ be a subset of an n-normed space $Y$. A mapping $S: M \rightarrow M$ belongs to the class $D(L, q)$ if for all $\mathrm{x}, \mathrm{y} \in \mathrm{M}, \mathrm{v} \in \mathrm{Y}^{\mathrm{n}-1}$ and $0<\mathrm{L}, \mathrm{q}<1$.

$$
\|S x-S y\|_{v} \leq L\|x-y\|_{v}+q\left[\|x-S x\|_{v}+\|y-S y\|_{v}\right]
$$

This paper consists of two sections. In section one, we introduce the concept of the stability on an n-normed space and prove that the Mann iteration scheme is stable under different types of mappings. In section two, we prove a data dependence result for a fixed point under a mapping that belongs to the class $D(L, q)$ with the help of the Mann iteration scheme.

\section{Notation:}

We will abbreviate $\mathrm{v}_{1}, \ldots, \mathrm{v}_{\mathrm{n}-1} \in \mathrm{Y}$ as $\mathrm{v}$ and $\left\|\mathrm{x}, \mathrm{v}_{1}, \ldots, \mathrm{v}_{\mathrm{n}-1}\right\|$ as $\|\mathrm{x}\|_{\mathrm{v}}$.

\section{\$1 Stability Results}

This section focuses on the stability of the Mann iteration scheme [8]. We define the Mann iteration as follows:

$$
\begin{gathered}
\mathrm{x}_{1} \in \mathrm{M} \\
\mathrm{x}_{\mathrm{k}+1}=\left(1-\alpha_{\mathrm{k}}\right) \mathrm{x}_{\mathrm{k}}+\alpha_{\mathrm{k}} S \mathrm{x}_{\mathrm{k}} \quad ; \mathrm{k} \in \mathbb{N}
\end{gathered}
$$

where $\left\{\alpha_{\mathrm{k}}\right\}$ is a sequence1satisfying $0<\alpha_{\mathrm{k}}<1$ for all $\mathrm{k} \in \mathbb{N}$ and $\sum_{\mathrm{k}=1}^{\infty} \alpha_{\mathrm{k}}=\infty$.

But before discussing the stability, we need to generalize the definition of the stability to the $n$-normed space.

\section{Definition 1.1:}

Let $\mathrm{Y}$ be an $\mathrm{n}$-normed space, $\mathrm{S}: \mathrm{Y} \rightarrow \mathrm{Y}$ is a mapping, and $\left\{\mathrm{x}_{\mathrm{k}}\right\} \subset \mathrm{Y}$ is the sequence generated by an iteration procedure

$$
\mathrm{x}_{\mathrm{k}+1}=\mathrm{f}\left(\mathrm{S}, \mathrm{x}_{\mathrm{k}}\right) ; \mathrm{k} \in \mathbb{N}
$$

where $x_{0} \in Y$. Suppose that $\left\{x_{k}\right\}$ converges to a fixed point $p$ of $S,\left\{y_{k}\right\}$ is an arbitrary sequence in $\mathrm{Y}$, and the set

$$
\varepsilon_{\mathrm{k}}=\left\|\mathrm{y}_{\mathrm{k}+1}-\mathrm{f}\left(\mathrm{S}, \mathrm{y}_{\mathrm{k}}\right)\right\|_{\mathrm{v}} ; \mathrm{k} \in \mathbb{N} \text {. }
$$

Then, the iteration (1) is said to be $S$ - Stable or stable with respect to $S$ if

$$
\lim _{\mathrm{k} \rightarrow \infty} \varepsilon_{\mathrm{k}}=0 \text { if and only if } \lim _{\mathrm{k} \rightarrow \infty}\left\|\mathrm{y}_{\mathrm{k}}-\mathrm{p}\right\|_{\mathrm{v}}=0 .
$$

The following lemma is essential to the main result of the section.

\section{Lemma 1.2 [9]:}

If $\delta$ is a real number such that $0 \leq \delta<1$, and $\left\{\varepsilon_{\mathrm{k}}\right\}$ is a sequence of positive numbers such that $\lim _{\mathrm{k} \rightarrow \infty} \varepsilon_{\mathrm{k}}=0$, then for any sequence of positive numbers $\left\{\mathrm{a}_{\mathrm{k}}\right\}$ satisfying

we have

$$
\mathrm{a}_{\mathrm{k}+1} \leq \delta \mathrm{a}_{\mathrm{k}}+\varepsilon_{\mathrm{k}} ; \quad \mathrm{k}=0,1,2, \ldots
$$

$$
\lim _{k \rightarrow \infty} a_{k}=0 \text {. }
$$

The next result is to prove that the Mann iteration scheme converges to a fixed point $\mathrm{p}$ of $\mathrm{S}$.

\section{Proposition 1.3:}

Let $\mathrm{M}$ be a convex subset of an $\mathrm{n}$-Banach space $\mathrm{Y}$ and a mapping $\mathrm{S}: \mathrm{M} \rightarrow \mathrm{M}$ be a mapping that belongs to the class $\mathrm{D}(\mathrm{L}, \mathrm{q})$ with $0<\mathrm{L}, \mathrm{q}<1, \mathrm{~L}+2 \mathrm{q}<1$. Then, the Mann iteration scheme converges to a fixed point $\mathrm{p}$ of $\mathrm{S}$.

\section{Proof.}

Using the Mann iteration scheme and since $S$ belongs to the class $D(L, q)$, we obtain that

$$
\begin{aligned}
\left\|x_{k+1}-p\right\|_{v} & =\left\|\left(1-\alpha_{k}\right) x_{k}+\alpha_{k} S x_{k}-p\right\|_{v} \\
& =\left\|\left(1-\alpha_{k}\right) x_{k}-\alpha_{k} p+\alpha_{k} p+\alpha_{k} S x_{k}-p\right\|_{v} \\
& =\left\|\left(1-\alpha_{k}\right) x_{k}-\left(1-\alpha_{k}\right) p+\alpha_{k} S x_{k}-\alpha_{k} p\right\|_{v}
\end{aligned}
$$


Note that:

$$
\begin{aligned}
& =\left\|\left(1-\alpha_{\mathrm{k}}\right)\left(\mathrm{x}_{\mathrm{k}}-\mathrm{p}\right)+\alpha_{\mathrm{k}}\left(S \mathrm{~S}_{\mathrm{k}}-\mathrm{p}\right)\right\|_{\mathrm{v}} \\
& \leq\left(1-\alpha_{\mathrm{k}}\right)\left\|\mathrm{x}_{\mathrm{k}}-\mathrm{p}\right\|_{\mathrm{v}}+\alpha_{\mathrm{k}}\left\|S \mathrm{x}_{\mathrm{k}}-\mathrm{p}\right\|_{\mathrm{v}} \\
& \leq\left(1-\alpha_{\mathrm{k}}\right)\left\|\mathrm{x}_{\mathrm{k}}-\mathrm{p}\right\|_{\mathrm{v}}+\alpha_{\mathrm{k}}\left(\mathrm{L}\left\|\mathrm{x}_{\mathrm{k}}-\mathrm{p}\right\|_{\mathrm{v}}\right. \\
& \left.\quad+\mathrm{q}\left(\left\|\mathrm{x}_{\mathrm{k}}-S \mathrm{x}_{\mathrm{k}}\right\|_{\mathrm{v}}+\|\mathrm{p}-\mathrm{Sp}\|_{\mathrm{v}}\right)\right) \\
& \quad=\left(1-\alpha_{\mathrm{k}}(1-\mathrm{L})\right)\left\|\mathrm{x}_{\mathrm{k}}-\mathrm{p}\right\|_{\mathrm{v}}+\alpha_{\mathrm{k}} \mathrm{q}\left\|\mathrm{x}_{\mathrm{k}}-S \mathrm{x}_{\mathrm{k}}\right\|_{\mathrm{v}}
\end{aligned}
$$

$\left\|\mathrm{x}_{\mathrm{k}}-\mathrm{Sx}_{\mathrm{k}}\right\|_{\mathrm{v}} \leq\left\|\mathrm{x}_{\mathrm{k}}-\mathrm{p}\right\|_{\mathrm{v}}+\left\|\mathrm{Sp}-\mathrm{S} \mathrm{x}_{\mathrm{k}}\right\|_{\mathrm{v}}$

Thus,

$$
\leq\left\|x_{k}-p\right\|_{v}+L\left\|x_{k}-p\right\|_{v}+q\left(\|S p-p\|_{v}+\left\|x_{k}-S x_{k}\right\|_{v}\right)
$$

By combining (1) and (2), we have

$$
\left\|\mathrm{x}_{\mathrm{k}}-\mathrm{S} \mathrm{x}_{\mathrm{k}}\right\|_{\mathrm{v}} \leq \frac{1+\mathrm{L}}{1-\mathrm{q}}\left\|\mathrm{x}_{\mathrm{k}}-\mathrm{p}\right\|_{\mathrm{v}}
$$

$\left\|\mathrm{x}_{\mathrm{k}+1}-\mathrm{p}\right\|_{\mathrm{v}} \leq\left(1-\alpha_{\mathrm{k}}(1-\mathrm{L})\right)\left\|\mathrm{x}_{\mathrm{k}}-\mathrm{p}\right\|_{\mathrm{v}}+\alpha_{\mathrm{k}} \mathrm{q}\left(\frac{1+\mathrm{L}}{1-\mathrm{q}}\right)\left\|\mathrm{x}_{\mathrm{k}}-\mathrm{p}\right\|_{\mathrm{v}}$

$$
\begin{aligned}
& =\left(1-\alpha_{\mathrm{k}}\left(1-\mathrm{L}-\mathrm{q}\left(\frac{1+\mathrm{L}}{1-\mathrm{q}}\right)\right)\right)\left\|\mathrm{x}_{\mathrm{k}}-\mathrm{p}\right\|_{\mathrm{v}} \\
& \quad \leq \quad \leq \prod_{\mathrm{k}=0}^{\mathrm{m}}\left(1-\alpha_{\mathrm{k}}\left(1-\mathrm{L}-\mathrm{q}\left(\frac{1+\mathrm{L}}{1-\mathrm{q}}\right)\right)\right)\left\|\mathrm{x}_{0}-\mathrm{p}\right\|_{\mathrm{v}}
\end{aligned}
$$

Observe that

$$
\prod_{\mathrm{k}=0}^{\mathrm{m}}\left(1-\alpha_{\mathrm{k}}\left(1-\mathrm{L}-\mathrm{q}\left(\frac{1+\mathrm{L}}{1-\mathrm{q}}\right)\right)\right) \leq \exp \left(-\sum_{\mathrm{k}=0}^{\mathrm{m}} \alpha_{\mathrm{k}}\left(1-\mathrm{L}-\mathrm{q}\left(\frac{1+\mathrm{L}}{1-\mathrm{q}}\right)\right)\right) \rightarrow 0 \text {, as } \mathrm{k} \rightarrow \infty
$$

From equation (3), we have

$$
\left\|\mathrm{x}_{\mathrm{k}+1}-\mathrm{p}\right\|_{\mathrm{v}} \leq\left\|\mathrm{x}_{0}-\mathrm{p}\right\|_{\mathrm{v}} \exp \left[\left(-\left(1-\mathrm{L}-\mathrm{q}\left(\frac{1+\mathrm{L}}{1-\mathrm{q}}\right)\right)\right) \sum_{\mathrm{k}=0}^{\mathrm{m}} \alpha_{\mathrm{k}}\right]
$$

Because $\sum_{\mathrm{k}=0}^{\mathrm{m}} \alpha_{\mathrm{k}}=\infty$ and taking the limit of both sides of inequality (4) yields $\lim _{\mathrm{n} \rightarrow \infty}\left\|\mathrm{x}_{\mathrm{k}+1}-\mathrm{p}\right\|_{\mathrm{v}}=0$.

Now, we discuss the stability for the Mann iteration scheme with respect to belongs to the class $\mathrm{D}(\mathrm{L}, \mathrm{q})$ mapping.

\section{Theorem 1.4:}

Let $Y$ be an $n$-normed space and $S: Y \rightarrow Y$ belongs to the class $D(L, q)$, where $0<L, q<1$. Suppose that $S$ has a fixed point $p$. For arbitrary $x_{0} \in Y$ with $L+2 q<1$, if there is $\lambda \in \mathbb{R}$, where $0<\lambda \leq$ $\alpha_{\mathrm{k}}<1$, then the Mann iteration scheme is S- stable.

\section{Proof.}

Let $\left\{\mathrm{y}_{\mathrm{k}}\right\} \subset \mathrm{Y}$ and define

Suppose that $\lim _{\mathrm{k} \rightarrow \infty} \varepsilon_{\mathrm{k}}=0$. Then

$$
\varepsilon_{\mathrm{k}}=\left\|\mathrm{y}_{\mathrm{k}+1}-\left(1-\alpha_{\mathrm{k}}\right) \mathrm{y}_{\mathrm{k}}-\alpha_{\mathrm{k}} S \mathrm{y}_{\mathrm{k}}\right\|_{\mathrm{v}}, \mathrm{k} \geq 0 .
$$

$\left\|\mathrm{y}_{\mathrm{k}+1}-\mathrm{p}\right\|_{\mathrm{v}} \leq\left\|\mathrm{y}_{\mathrm{k}+1}-\left(1-\alpha_{\mathrm{k}}\right) \mathrm{y}_{\mathrm{k}}-\alpha_{\mathrm{k}} S \mathrm{y}_{\mathrm{k}}\right\|_{\mathrm{v}}$

$$
+\left\|\left(1-\alpha_{\mathrm{k}}\right) \mathrm{y}_{\mathrm{k}}+\alpha_{\mathrm{k}} S \mathrm{y}_{\mathrm{k}}-\mathrm{p}\right\|_{\mathrm{v}}
$$

$$
\begin{aligned}
& =\varepsilon_{\mathrm{k}}+\left\|\left(1-\alpha_{\mathrm{k}}\right) \mathrm{y}_{\mathrm{k}}+\alpha_{\mathrm{k}} \mathrm{p}-\alpha_{\mathrm{k}} \mathrm{p}+\alpha_{\mathrm{k}} S y_{\mathrm{k}}-\mathrm{p}\right\|_{\mathrm{v}} \\
& =\varepsilon_{\mathrm{k}}+\left\|\left(1-\alpha_{\mathrm{k}}\right) \mathrm{y}_{\mathrm{k}}-\left(1-\alpha_{\mathrm{k}}\right) \mathrm{p}+\alpha_{\mathrm{k}} S y_{\mathrm{k}}-\alpha_{\mathrm{k}} \mathrm{p}\right\|_{\mathrm{v}} \\
& =\varepsilon_{\mathrm{k}}+\left\|\left(1-\alpha_{\mathrm{k}}\right)\left(\mathrm{y}_{\mathrm{k}}-\mathrm{p}\right)+\alpha_{\mathrm{k}}\left(S y_{\mathrm{k}}-\mathrm{p}\right)\right\|_{\mathrm{v}} \\
& \leq \varepsilon_{\mathrm{k}}+\left(1-\alpha_{\mathrm{k}}\right)\left\|\mathrm{y}_{\mathrm{k}}-\mathrm{p}\right\|_{\mathrm{v}}+\alpha_{\mathrm{k}}\left\|S y_{\mathrm{k}}-\mathrm{Sp}\right\|_{\mathrm{v}}
\end{aligned}
$$

Since $S$ a belongs to the class $D(L, q)$, we have

$\left\|y_{k+1}-p\right\|_{v} \leq \varepsilon_{k}+\left(1-\alpha_{k}\right)\left\|y_{k}-p\right\|_{v}+\alpha_{k}\left[L\left\|y_{k}-p\right\|_{v}\right.$

$$
\left.+\mathrm{q}\left(\left\|\mathrm{y}_{\mathrm{k}}-S \mathrm{y}_{\mathrm{k}}\right\|_{\mathrm{v}}+\|\mathrm{p}-\mathrm{Sp}\|_{\mathrm{v}}\right)\right]
$$

From proposition (1.4) equation (2), we have

$$
\begin{aligned}
\left\|y_{\mathrm{k}+1}-\mathrm{p}\right\|_{\mathrm{v}} \leq & \varepsilon_{\mathrm{k}}+\left(1-\alpha_{\mathrm{k}}\right)\left\|\mathrm{y}_{\mathrm{k}}-\mathrm{p}\right\|_{\mathrm{v}} \\
& +\alpha_{\mathrm{k}}\left[\mathrm{L}\left\|\mathrm{y}_{\mathrm{k}}-\mathrm{p}\right\|_{\mathrm{v}}+\mathrm{q}\left(\frac{1+\mathrm{L}}{1-\mathrm{q}}\right)\left\|\mathrm{y}_{\mathrm{k}}-\mathrm{p}\right\|_{\mathrm{v}}\right] \\
& \left(1-\alpha_{\mathrm{k}}\left(1-\mathrm{L}-\mathrm{q}\left(\frac{1+\mathrm{L}}{1-\mathrm{q}}\right)\right)\right)\left\|\mathrm{y}_{\mathrm{k}}-\mathrm{p}\right\|_{\mathrm{v}}+\varepsilon_{\mathrm{k}} .
\end{aligned}
$$


Therefore

$$
\left\|\mathrm{y}_{\mathrm{k}+1}-\mathrm{p}\right\|_{\mathrm{v}} \leq\left(1-\lambda\left(1-\left(\frac{\mathrm{L}+\mathrm{q}}{1-\mathrm{q}}\right)\right)\right)\left\|\mathrm{y}_{\mathrm{k}}-\mathrm{p}\right\|_{\mathrm{v}}+\varepsilon_{\mathrm{k}}
$$

Since $L+2 q<1$ and $0<1-\lambda\left(1-\left(\frac{\mathrm{L}+\mathrm{q}}{1-\mathrm{q}}\right)\right)<1$, by using Lemma in (1.2), we get

$$
\lim _{\mathrm{n} \rightarrow \infty}\left\|\mathrm{y}_{\mathrm{k}}-\mathrm{p}\right\|_{\mathrm{v}}=0
$$

Conversely, Let $\lim _{\mathrm{n} \rightarrow \infty}\left\|\mathrm{y}_{\mathrm{k}}-\mathrm{p}\right\|_{\mathrm{v}}=0$. To show that $\lim _{\mathrm{k} \rightarrow \infty} \varepsilon_{\mathrm{k}}=0$, then

$\varepsilon_{\mathrm{k}}=\left\|\mathrm{y}_{\mathrm{k}+1}-\left(1-\alpha_{\mathrm{k}}\right) \mathrm{y}_{\mathrm{k}}-\alpha_{\mathrm{k}} \mathrm{Sy}_{\mathrm{k}}\right\|_{\mathrm{v}}$

$$
\leq\left\|y_{k+1}-p\right\|_{v}+\left(1-\alpha_{k}\right)\left\|y_{k}-p\right\|_{v}+\alpha_{k}\left\|S y_{k}-p\right\|_{v}
$$

By using the definition that belongs to the class $\mathrm{D}(\mathrm{L}, \mathrm{q})$, we have

$\varepsilon_{\mathrm{k}} \leq\left\|\mathrm{y}_{\mathrm{k}+1}-\mathrm{p}\right\|_{\mathrm{v}}+\left(1-\alpha_{\mathrm{k}}\right)\left\|\mathrm{y}_{\mathrm{k}}-\mathrm{p}\right\|_{\mathrm{v}}$

$$
+\alpha_{\mathrm{k}}\left[\mathrm{L}\left\|\mathrm{y}_{\mathrm{k}}-\mathrm{p}\right\|_{\mathrm{v}}+\mathrm{q}\left(\left\|\mathrm{y}_{\mathrm{k}}-\mathrm{Sy}_{\mathrm{k}}\right\|_{\mathrm{v}}+\|\mathrm{p}-\mathrm{Sp}\|_{\mathrm{v}}\right)\right]
$$

From the equation (2) of the proposition (1.3), we have

$$
\varepsilon_{\mathrm{k}} \leq\left\|\mathrm{y}_{\mathrm{k}+1}-\mathrm{p}\right\|_{\mathrm{v}}+\left(1-\alpha_{\mathrm{k}}\left(1-\left(\frac{\mathrm{L}+\mathrm{q}}{1-\mathrm{q}}\right)\right)\left\|\mathrm{y}_{\mathrm{k}}-\mathrm{p}\right\|_{\mathrm{v}}\right.
$$

Then, $\varepsilon_{\mathrm{k}} \rightarrow 0$ as $\mathrm{k} \rightarrow \infty$.

\section{\$2 Data Dependence Results}

In some cases, to compute a fixed point $p$ of $S$ we use a certain approximate operator $\widetilde{S}$ of $S$ to approximate a fixed point $\tilde{p}$ of $\tilde{S}$. So the natural question arises: Does $\tilde{p}$ approximate $p$ ? If yes, how can we compute $\|\mathrm{p}-\tilde{\mathrm{p}}\|_{\mathrm{v}}$ ?

In this section, we try to answer this question. But first we introduce the definition of approximate mapping and lemma.

\section{Definition 2.1:}

Let $\mathrm{Y}$ be an $\mathrm{n}$-normed space and $\mathrm{S}$ and $\tilde{\mathrm{S}}$ be two mappings. We say that $\tilde{\mathrm{S}}$ is an approximate mapping of $S$, if for all $\mathrm{x} \in \mathrm{Y}$ and for fixed $\epsilon>0$ we have:

\section{Lemma 2.2[7]:}

$$
\|\mathrm{Sx}-\tilde{\mathrm{S} x}\|_{\mathrm{v}} \leq \epsilon .
$$

Let $\left\{\mathrm{a}_{\mathrm{k}}\right\}$ be a nonnegative sequence for which one assumes that there exists $\mathrm{k}_{0} \in \mathbb{N}$, such that for all $\mathrm{k} \in \mathbb{N} ; \mathrm{k} \geq \mathrm{k}_{0}$ one has satisfied the inequality

$$
\mathrm{a}_{\mathrm{k}+1} \leq\left(1-\delta_{\mathrm{k}}\right) \mathrm{a}_{\mathrm{k}}+\delta_{\mathrm{k}} \sigma_{\mathrm{k}}
$$

where $\delta_{\mathrm{k}} \in(0,1), \sum_{\mathrm{n}=1}^{\infty} \delta_{\mathrm{k}}=\infty$ and $\sigma_{\mathrm{k}} \geq 0$, then the following inequality holds

$$
0 \leq \lim _{\mathrm{n} \rightarrow \infty} \sup \mathrm{a}_{\mathrm{k}} \leq \limsup _{\mathrm{n} \rightarrow \infty} \sigma_{\mathrm{k}}
$$

Now, we can establish the following data dependence result.

\section{Theorem 2.3:}

Let $\mathrm{Y}$ be an $\mathrm{n}$-Banach space, $\mathrm{M}$ be a nonempty closed convex subset of $\mathrm{Y}, \mathrm{S}: \mathrm{M} \rightarrow \mathrm{M}$ belongs to the class $D(L, q)$ operator with the fixed point $p$, and $\tilde{S}: M \rightarrow M$ is a mapping with a fixed point $\tilde{p}$. If the following relation is satisfied for all $\mathrm{x} \in \mathrm{M}$ :

$$
\|\mathrm{Sx}-\widetilde{\mathrm{S}} \mathrm{x}\|_{\mathrm{v}} \leq \epsilon
$$

then, for $\epsilon>0$ and $\mathrm{L}, \mathrm{q} \in(0,1), \mathrm{L}+2 \mathrm{q}<1$, we have

\section{Proof.}

$$
\|p-\tilde{p}\|_{v} \leq \frac{\epsilon}{1-\frac{L+q}{1-q}}
$$

$$
\begin{aligned}
& \text { Note that, } \\
& \begin{aligned}
\left\|\widetilde{\mathrm{x}}_{\mathrm{k}}-\mathrm{S} \widetilde{\mathrm{x}}_{\mathrm{k}}\right\|_{\mathrm{v}} & \leq\left\|\widetilde{\mathrm{x}}_{\mathrm{k}}-\mathrm{p}\right\|_{\mathrm{v}}+\left\|\mathrm{Sp}-\mathrm{S} \widetilde{\mathrm{x}}_{\mathrm{k}}\right\|_{\mathrm{v}} \\
& \leq\left\|\widetilde{\mathrm{x}}_{\mathrm{k}}-\mathrm{p}\right\|_{\mathrm{v}}+\mathrm{L}\left\|\mathrm{p}-\widetilde{\mathrm{x}}_{\mathrm{k}}\right\|_{\mathrm{v}}+\mathrm{q}\left(\|\mathrm{p}-\mathrm{Sp}\|_{\mathrm{v}}+\left\|\widetilde{\mathrm{x}}_{\mathrm{k}}-\mathrm{S} \widetilde{\mathrm{x}}_{\mathrm{n}}\right\|_{\mathrm{v}}\right) \\
& \leq(1+\mathrm{L})\left(\left\|\widetilde{\mathrm{x}}_{\mathrm{k}}-\mathrm{x}_{\mathrm{k}}\right\|_{\mathrm{v}}+\left\|\mathrm{x}_{\mathrm{k}}-\mathrm{p}\right\|_{\mathrm{v}}\right)+\mathrm{q}\left\|\widetilde{\mathrm{x}}_{\mathrm{k}}-\mathrm{S} \widetilde{\mathrm{x}}_{\mathrm{n}}\right\|_{\mathrm{v}}
\end{aligned}
\end{aligned}
$$

Thus,

And similarly

$$
\left\|\widetilde{\mathrm{x}}_{\mathrm{k}}-\mathrm{S} \widetilde{\mathrm{x}}_{\mathrm{k}}\right\|_{\mathrm{v}} \leq \frac{1+\mathrm{L}}{1-\mathrm{q}}\left\|\widetilde{\mathrm{x}}_{\mathrm{k}}-\mathrm{x}_{\mathrm{k}}\right\|_{\mathrm{v}}+\frac{1+\mathrm{L}}{1-\mathrm{q}}\left\|\mathrm{x}_{\mathrm{k}}-\mathrm{p}\right\|_{\mathrm{v}}
$$

$$
\left\|\mathrm{x}_{\mathrm{k}}-\mathrm{Sx}_{\mathrm{k}}\right\|_{\mathrm{v}} \leq \frac{1+\mathrm{L}}{1-\mathrm{q}}\left\|\mathrm{x}_{\mathrm{k}}-\mathrm{p}\right\|_{\mathrm{v}}
$$


By using the Mann iteration scheme, we have

$$
\begin{aligned}
\left\|\mathrm{x}_{\mathrm{k}+1}-\tilde{\mathrm{x}}_{\mathrm{k}+1}\right\|_{\mathrm{v}} & =\left\|\left(1-\alpha_{\mathrm{k}}\right) \mathrm{x}_{\mathrm{k}}+\alpha_{\mathrm{n}} S \mathrm{x}_{\mathrm{k}}-\left(1-\alpha_{\mathrm{k}}\right) \tilde{\mathrm{x}}_{\mathrm{k}}-\alpha_{\mathrm{k}} \tilde{\mathrm{S}}_{\mathrm{x}}\right\|_{\mathrm{v}} \\
& \leq\left(1-\alpha_{\mathrm{k}}\right)\left\|\mathrm{x}_{\mathrm{k}}-\tilde{\mathrm{x}}_{\mathrm{k}}\right\|_{\mathrm{v}}+\alpha_{\mathrm{k}}\left\|S \mathrm{x}_{\mathrm{k}}-\tilde{\mathrm{S}} \mathrm{x}_{\mathrm{k}}\right\|_{\mathrm{v}}+\alpha_{\mathrm{k}}\left\|\tilde{\mathrm{S}} \mathrm{x}_{\mathrm{k}}-\tilde{\mathrm{S}}_{\mathrm{k}}\right\|_{\mathrm{v}} \\
& \leq \alpha_{\mathrm{k}} \epsilon+\left(1-\alpha_{\mathrm{k}}\right)\left\|\mathrm{x}_{\mathrm{k}}-\tilde{\mathrm{x}}_{\mathrm{k}}\right\|_{\mathrm{v}}+\alpha_{\mathrm{k}} \mathrm{L}\left\|\mathrm{x}_{\mathrm{k}}-\tilde{\mathrm{x}}_{\mathrm{k}}\right\|_{\mathrm{v}} \\
& +\alpha_{\mathrm{k}} \mathrm{q}\left(\left\|\mathrm{x}_{\mathrm{k}}-S \mathrm{x}_{\mathrm{k}}\right\|_{\mathrm{v}}+\left\|\widetilde{\mathrm{x}}_{\mathrm{k}}-\mathrm{S} \tilde{\mathrm{x}}_{\mathrm{k}}\right\|_{\mathrm{v}}\right)
\end{aligned}
$$

By combining (1), (2) and (3), we have

$$
\begin{aligned}
\left\|\mathrm{x}_{\mathrm{k}+1}-\tilde{\mathrm{x}}_{\mathrm{k}+1}\right\|_{\mathrm{v}} \leq & \left(1-\alpha_{\mathrm{k}}\right)\left\|\mathrm{x}_{\mathrm{k}}-\tilde{\mathrm{x}}_{\mathrm{k}}\right\|_{\mathrm{v}}+\alpha_{\mathrm{k}} \epsilon+\alpha_{\mathrm{k}} \mathrm{L}\left\|\mathrm{x}_{\mathrm{k}}-\tilde{\mathrm{x}}_{\mathrm{k}}\right\|_{\mathrm{v}} \\
+ & \alpha_{\mathrm{k}} \mathrm{q}\left(\frac{1+\mathrm{L}}{1-\mathrm{q}}\left\|\mathrm{x}_{\mathrm{k}}-\mathrm{p}\right\|_{\mathrm{v}}+\frac{1+\mathrm{L}}{1-\mathrm{q}}\left\|\widetilde{\mathrm{x}}_{\mathrm{k}}-\mathrm{x}_{\mathrm{k}}\right\|_{\mathrm{v}}+\frac{1+\mathrm{a}}{1-\mathrm{b}}\left\|\mathrm{x}_{\mathrm{k}}-\mathrm{p}\right\|_{\mathrm{v}}\right) \\
= & \left(1-\alpha_{\mathrm{k}}\left(1-\mathrm{L}-\mathrm{q}\left(\frac{1+\mathrm{L}}{1-\mathrm{q}}\right)\right)\right)\left\|\mathrm{x}_{\mathrm{k}}-\tilde{\mathrm{x}}_{\mathrm{k}}\right\|_{\mathrm{v}} \\
& +2 \alpha_{\mathrm{k}} \mathrm{q}\left(\frac{1+\mathrm{L}}{1-\mathrm{q}}\right)\left\|\mathrm{x}_{\mathrm{k}}-\mathrm{p}\right\|_{\mathrm{v}}+\alpha_{\mathrm{k}} \epsilon \\
= & \left(1-\alpha_{\mathrm{k}}\left(1-\frac{\mathrm{L}+\mathrm{q}}{1-\mathrm{q}}\right)\right)\left\|\mathrm{x}_{\mathrm{k}}-\tilde{\mathrm{x}}_{\mathrm{k}}\right\|_{\mathrm{v}} \\
& +\alpha_{\mathrm{k}}\left(1-\frac{\mathrm{L}+\mathrm{q}}{1-\mathrm{q}}\right) \frac{2 \mathrm{q} \frac{1+\mathrm{L}}{1-\mathrm{q}}\left\|\mathrm{x}_{\mathrm{k}}-\mathrm{p}\right\|_{\mathrm{v}}+\epsilon}{1-\frac{\mathrm{L}+\mathrm{q}}{1-\mathrm{q}}}
\end{aligned}
$$

By putting:

$$
\begin{gathered}
\mathrm{a}_{\mathrm{k}}=\left\|\mathrm{x}_{\mathrm{k}}-\tilde{\mathrm{x}}_{\mathrm{k}}\right\|_{\mathrm{v}}, \text { since } \mathrm{L}+2 \mathrm{q}<1 \text { thus, } \delta_{\mathrm{k}}=\alpha_{\mathrm{k}}\left(1-\frac{\mathrm{L}+\mathrm{q}}{1-\mathrm{q}}\right) \in(0,1), \text { and } \\
\sigma_{\mathrm{k}}=\frac{2 \mathrm{q} \frac{1+\mathrm{L}}{1-\mathrm{q}}\left\|\mathrm{x}_{\mathrm{k}}-\mathrm{p}\right\|_{\mathrm{v}}+\epsilon}{1-\frac{\mathrm{L}+\mathrm{q}}{1-\mathrm{q}}}
\end{gathered}
$$

From lemma ( 2.2 ), we obtain:

$$
0 \leq \lim _{n \rightarrow \infty} \sup \left\|x_{k}-\tilde{x}_{k}\right\|_{v} \leq \lim _{k \rightarrow \infty} \sup \frac{2 q \frac{1+L}{1-q}\left\|x_{k}-p\right\|_{v}+\epsilon}{1-\frac{L+q}{1-q}}
$$

From proposition (1.3) $\lim _{\mathrm{k} \rightarrow \infty}\left\|\mathrm{x}_{\mathrm{k}}-\mathrm{p}\right\|_{\mathrm{v}}=0$ and $\mathrm{Sp}=\mathrm{p}$, therefore

$$
\|\mathrm{p}-\tilde{\mathrm{p}}\|_{\mathrm{v}} \leq \frac{\epsilon}{1-\frac{\mathrm{L}+\mathrm{q}}{1-\mathrm{q}}} \text {. }
$$

\section{References}

1. Gunawan H. and Mashadi M. 2001. On n-normed spaces, Int. J. Math. Sci. 27(10): 631-639.

2. Harder A. M. 1987. Fixed Point Theory and Stability Results for Fixed Point Iteration Procedures, $\mathrm{Ph}$. D. thesis University of Missouri-Rolla, Missouri,.

3. Harder A. M. and Hicks T. L. 1988. Stability Results for Fixed Point Iteration Procedures, Math. Japon. 33: 693-706.

4. Şoltuz S.M. 2001. Data Dependence for Mann iteration. Octogon Math. Magazine, 9: 825-828.

5. Rus I. A. and Muresan S. 1998. Data Dependence of The Fixed Points Set of Weakly Picard Operators. Stud. Univ. Babes-Bolyai, 43 : 79-83.

6. Espiýnola R. and Petrusel A. 2005. Existence And Data Dependence of Fixed Points for Multi Valued Operators on Gauge Spaces. J.Math. Anal. Apple. 309: 420-432.

7. Soltuz S.M. and Grosan T. 2008. Data Dependence for Ishikawa Iteration When Dealing With Contractive-Like Operators. Fixed Point Theory Appl. Article ID 242916. doi:10.1155 /2008 1242916.

8. Hamed M. and Jamil Z. 2019. Mann Iteration Processes on Uniform Convex n-Banach space. Iraqi Journal of Science, 60(7): 1603-1608

9. Berinde V. 2002. On The Stability of Some Fixed Point Procedures, Bull. Stiint. Univ. Baia Mare, Ser. B, Matematica-Informatica, XVIII(1): 7-14. 